\title{
A Case of Fever and Erythema Nodosum-Like Lesions Leading to a New Diagnosis of Gamma-Delta T-Cell Lymphoma Complicated by Hemophagocytic Lymphohistiocytosis
}

\author{
William S. Murphy ${ }^{a} \quad$ Jennifer E. Yeh ${ }^{b}$ Rosalynn M. Nazarian ${ }^{c}$ \\ Aliyah R. Sohanic ${ }^{c}$ Joseph Kvedard Daniela Kroshinsky ${ }^{d}$ \\ a Harvard Medical School, Harvard Business School, Boston, MA, USA; ${ }^{b}$ Harvard \\ Combined Dermatology Residency Program, Boston, MA, USA; ${ }^{C}$ Department of Pathology, \\ Massachusetts General Hospital, Boston, MA, USA; ${ }^{d}$ Department of Dermatology, \\ Massachusetts General Hospital, Boston, MA, USA
}

\section{Keywords}

Cutaneous T-cell lymphoma - Gamma-delta T-cell lymphoma - Erythema nodosum mimic . Hemophagocytic lymphohistiocytosis

\begin{abstract}
A 69-year-old Vietnamese female presented with fever and new-onset tender subcutaneous nodules on her trunk and lower extremities initially thought to be clinically consistent with erythema nodosum. A biopsy showed an atypical, predominantly lobular lymphocytic panniculitis with admixed neutrophils, karyorrhectic debris, and histiocytes with subcutaneous fat necrosis. Immunohistochemistry was consistent with gamma-delta T-cell lymphoma. The patient was initiated on a chemotherapy regimen of cyclophosphamide, doxorubicin, vincristine, etoposide, and prednisone (CHOEP) with partial remission, and is currently undergoing evaluation for bone marrow transplant. This case highlights the ability of cutaneous gamma-delta T-cell lymphoma to mimic more common cutaneous conditions such as erythema nodosum, and stresses the importance of a broad differential for new presentation of tender subcutaneous nodules with concomitant systemic symptoms.



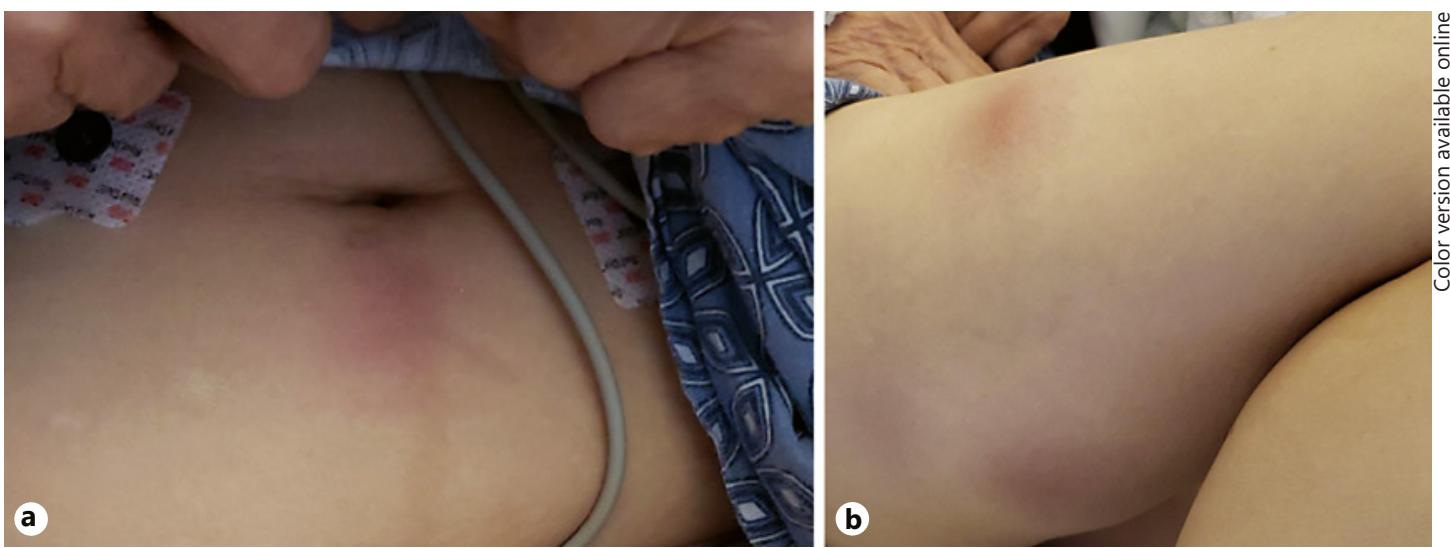

Fig. 1. a Representative tender erythematous subcutaneous nodule on the lower abdomen. b Representative tender violaceous subcutaneous nodules on the lower right extremity.

\section{Case Report}

A 69-year-old Vietnamese female with a history of thyroid cancer status post thyroidectomy and untreated chronic hepatitis B presented with 10 days of fever, malaise, and painful skin nodules. Three weeks prior to presentation, she had developed a tender violaceous lesion on her left thigh. At that time, the patient presented to her primary care physician who evaluated for deep venous thrombosis, which was negative, and also administered the herpes zoster vaccine. She subsequently developed fever, flu-like symptoms, and multiple similar nodules on her abdomen and lower extremities, prompting presentation to the hospital. There, she underwent a comprehensive infectious workup including cultures, respiratory viral panel, hepatitis serologies, HIV, tuberculosis, and Lyme disease, which revealed her known chronic hepatitis B infection but was otherwise negative. She received broadspectrum antibiotics; however, she continued to spike fevers to $103.6 \mathrm{~F}$. Clinically, her nodules were thought to be most consistent with erythema nodosum. Dermatology was consulted and found pink, blanching, tender subcutaneous firm nodules on the bilateral lower extremities (Fig. 1). A punch biopsy was performed.

Histopathologic examination of a representative lesion showed an atypical, predominantly lobular lymphocytic panniculitis with admixed neutrophils with karyorrhectic debris and histiocytes with subcutaneous fat necrosis. The overlying skin showed a vacuolar interface dermatitis, although no significant infiltrate was seen within the dermis. Initial diagnostic considerations included an infectious etiology, connective tissue disorder, subcutaneous panniculitis-like T-cell lymphoma, and gamma-delta T-cell lymphoma. Special stains (GMS, PAS/D, AFB, Fite, and Brown-Hopps) were negative for microorganisms. Immunohistochemical stains revealed CD3+ CD2+ lymphocytes with dim CD5 expression. A subset of the CD3+ cells rimming subcutaneous adipocytes stained positive for CD8 and CD56, and negative for CD4. Ki67 showed a high proliferation index in these cells. CD123 stain showed no significant plasmacytoid dendritic cell aggregates. The majority of the CD3+ T-cell rimming adipocytes in the panniculus were highlighted by perforin and TCR delta immunohistochemistry and showed negative staining for Beta F1 (Fig. 2). CD30 and EBER stains were negative in tumor cells. Taken together, the histopathologic and immunohistochemical findings were most consistent with cutaneous involvement by gamma-delta T-cell lymphoma with patchy CD8+ staining and subcutaneous localization. 
Fig. 2. Cutaneous involvement by gamma-delta T-cell lymphoma. a Punch biopsy shows a predominantly lobular panniculitis (H\&E. $\times 2$ ). b A vacuolar interface dermatitis is present $($ H\&E. $\times 20)$. c An atypical lobular lymphoid infiltrate is seen with adipocyte rimming in the subcutis (H\&E. $\times 20$ ). d Immunohistochemistry for CD3 reveals a prominent T-cell lymphoid infiltrate (CD3 stain, $\times 20$ ). e CD2 expression is retained in the CD3+ T-cells (CD2 stain, $\times 20$ ). $\mathbf{f}$ CD5 expression is dim in the CD3+ T-cells (CD5 stain, $\times 20 \times)$. g Very rare CD4+ Tcells are present (CD4 stain, $\times 20$ ). h Immunohistochemical stain for CD8 highlights many atypical lymphocytes, including those rimming adipocytes, but not all CD3+ $\mathrm{T}$ cells stain positive (CD8 stain, $\times 20$ ). i Ki67 immunohistochemistry reveals a high proliferation index in the atypical cells (Ki67 stain, $\times 20$ ). j No significant plasmacytoid dendritic cell aggregates are identified by CD123 (CD123 stain, $\times 20$ ). $\mathbf{k}$ The atypical cells are strongly positive for $\mathrm{T}$ cell receptor (TCR) delta by immunohistochemistry (TCR delta stain $\times 20$ ). I The atypical cells are negative for Beta F1 (Beta F1 stain, $\times 20$ ).




Given these biopsy results, the patient underwent a positron emission computed tomography, which showed FDG uptake corresponding to subcutaneous lesions along the abdominal wall, inguinal regions, and thighs. On day 4 of her hospitalization, she developed a new transaminitis (aspartate aminotransferase $752 \mathrm{U} / \mathrm{L}$ and alanine aminotransferase $333 \mathrm{U} / \mathrm{L}$ ) thought to be consistent with hemophagocytic lymphohistiocytosis. The patient was started on prednisone, then initiated on a chemotherapy regimen of cyclophosphamide, doxorubicin, vincristine, etoposide, and prednisone (CHOEP). She tolerated the chemotherapy well, and on repeat positron emission computed tomography was noted to have partial remission of the previously noted FDG-avid lesions. Her transaminitis resolved over the course of treatment. The patient is currently undergoing evaluation for allogeneic stem cell transplant (SCT).

\section{Discussion}

Cutaneous gamma-delta T-cell lymphoma (CGDTCL) is very rare, representing less than $1 \%$ of all primary cutaneous lymphomas [1]. The pathogenesis is unclear, although it is thought to be related to chronic antigenic stimulation [2]. The disease presents with indurated plaques or nodules on the extremities, and is often mistaken for arthropod bites, cellulitis, pyoderma, or as in this case, erythema nodosum [3]. The ability of CGDTCL to mimic many common diseases likely contributes to its significant underdiagnosis [3].

CGDTCL was recognized as a distinct entity by the World Health Organization in 2008 [4]. Guitart et al. [3] reviewed a multicenter case series of patients to determine characteristics of the disease. CGDTCL most commonly involved the lower extremities (75\% of the patients), torso (57\%), arms (53\%), and head and neck (26\%). Over time, 51\% of the patients' lesions ulcerated. Most patients reported B symptoms (54\%), the median age was 61 years, and there was no gender predilection [3]. Additionally, the most frequent comorbidities included autoimmunity (23\%), other lymphoma or leukemia (9\%), carcinomas (8\%), and viral hepatitis (4\%) [3]. Concurrent hemophagocytic syndrome, as seen in our patient, was common and portended a worse prognosis $[2,5]$.

CGDTCL is diagnosed by skin biopsy, which typically shows epidermotropic, dermal, and subcutaneous involvement [6]. Frequently, multiple histologic patterns may be seen in a single patient or biopsy [6]. As in our patient, rimming of fat cells is frequently seen in cases with subcutaneous involvement [6]. These histopathologic features, in combination with immunohistochemical stains, are helpful to differentiate CGDTCL from subcutaneous panniculitis-like T-cell lymphoma, which is a neoplasm of alpha/beta $\mathrm{T}$ cells generally confined to the subcutaneous tissue $[1,7,8]$.

The prognosis of CGDTCL is poor compared to other cutaneous lymphomas, with a reported median survival of 15 months [5, 9]. First-line treatment is doxorubicin-containing polychemotherapy, with SCT reserved for progressive disease [1]. A recent case series of 7 patients with CGDTCL and subcutaneous panniculitis-like T-cell lymphoma showed that $4 / 7$ (57\%) patients who underwent allogenic SCT were disease-free at a median follow-up of 5.6 years, and 2/2 (100\%) patients undergoing autologous SCT were disease-free at a median follow-up of 1.91 years [10].

Although our patient presented with findings initially felt to be clinically consistent with erythema nodosum, skin biopsy led to the diagnosis of new-onset primary CGDTCL. Consistent with published characteristics of patients with CGDTCL, our patient presented in her sixties with lesions on her lower extremities and trunk, and had untreated viral hepatitis. She also reported fever and body aches, and was found to have a new transaminitis, consistent with hemophagocytic lymphohistiocytosis. The rapid identification of her CGDTCL allowed for prompt treatment with steroids and chemotherapy, leading to her current partial remission 
and consideration for SCT for definitive treatment. This case highlights the importance of maintaining a broad differential for a new presentation with erythematous nodules and obtaining confirmatory biopsies where appropriate.

\section{Statement of Ethics}

The authors have no ethical conflicts to disclose. Patient consent was not required for this study, given the nonidentifiability of the patient.

\section{Disclosure Statement}

The authors have no conflicts of interest to declare.

\section{Author Contributions}

W.S.M. and J.E.Y. wrote the manuscript.

R.M.N. and A.R.S. performed and interpreted histopathology results and reviewed the manuscript.

J.K. and D.K. provided extensive clinical expertise and reviewed the manuscript.

\section{References}

1 Foppoli M, Ferreri AJ. Gamma-delta t-cell lymphomas. Eur J Haematol. 2015 Mar;94(3):206-18.

2 Tripodo C, Iannitto E, Florena AM, Pucillo CE, Piccaluga PP, Franco V, et al. Gamma-delta T-cell lymphomas. Nat Rev Clin Oncol. 2009 Dec;6(12):707-17.

3 Guitart J, Weisenburger DD, Subtil A, Kim E, Wood G, Duvic M, et al. Cutaneous $\gamma \delta$ T-cell lymphomas: a spectrum of presentations with overlap with other cytotoxic lymphomas. Am J Surg Pathol. 2012 Nov;36(11):1656-65.

4 Jaffe ES. The 2008 WHO classification of lymphomas: implications for clinical practice and translational research. Hematology (Am Soc Hematol Educ Program). 2009;2009(1):523-31.

5 Toro JR, Liewehr DJ, Pabby N, Sorbara L, Raffeld M, Steinberg SM, et al. Gamma-delta T-cell phenotype is associated with significantly decreased survival in cutaneous T-cell lymphoma. Blood. 2003 May;101(9):3407-12.

6 Willemze R, Jaffe ES, Burg G, Cerroni L, Berti E, Swerdlow SH, et al. WHO-EORTC classification for cutaneous lymphomas. Blood. 2005 May;105(10):3768-85.

7 Kong YY, Dai B, Kong JC, Zhou XY, Lu HF, Shen L, et al. Subcutaneous panniculitis-like T-cell lymphoma: a clinicopathologic, immunophenotypic, and molecular study of 22 Asian cases according to WHO-EORTC classification. Am J Surg Pathol. 2008 Oct;32(10):1495-502.

8 Willemze R, Jansen PM, Cerroni L, Berti E, Santucci M, Assaf C, et al.; EORTC Cutaneous Lymphoma Group. Subcutaneous panniculitis-like T-cell lymphoma: definition, classification, and prognostic factors: an EORTC Cutaneous Lymphoma Group Study of 83 cases. Blood. 2008 Jan;111(2):838-45.

9 Go RS, Wester SM. Immunophenotypic and molecular features, clinical outcomes, treatments, and prognostic factors associated with subcutaneous panniculitis-like T-cell lymphoma: a systematic analysis of 156 patients reported in the literature. Cancer. 2004 Sep;101(6):1404-13.

10 Gibson JF, Alpdogan O, Subtil A, Girardi M, Wilson LD, Roberts K, et al. Hematopoietic stem cell transplantation for primary cutaneous $\gamma \delta$ T-cell lymphoma and refractory subcutaneous panniculitis-like T-cell lymphoma. J Am Acad Dermatol. 2015 Jun; 72(6):1010-5.e5. 